\title{
PORCENTAJE DE HEMBRAS DEL GUACAMAYO CABECIAZUL Primolius couloni (Sclater, 1876) MANTENIDOS EN CAUTIVERIO EN CUATRO CENTROS DE MANEJO DE FAUNA SILVESTRE EN EL PERÚ
}

\section{FEMALES' PERCENTAGE OF THE BLUEHEADED MACAW Primolius couloni (Sclater, 1876) KEPT IN CAPTIVITY IN FOUR WILDLIFE MANAGEMENT CENTRES IN PERU}

\author{
Aimy Cáceres ${ }^{1}$ y Martha Williams de Castro²
}

\begin{abstract}
Resumen
Se evaluó un total de 46 individuos de Primolius couloni en cuatro centros de manejo de fauna silvestre del Perú: Parque Zoológico Huachipa, Zoocriadero CIRES-Perú, Zoocriadero Jean De Conick y Zoocriadero Mundo Silvestre. El objetivo del estudio fue determinar el porcentaje de hembras de Primolius couloni mantenidas en cautiverio, con el fin de ayudar a los centros de manejo que los albergan a implementar futuros planes de manejo reproductivo. Los resultados muestran que el porcentaje de hembras de Primolius couloni fue tan sólo de $10.26 \%$, es decir tan sólo hay cuatro hembras de los 39 individuos sexados exitosamente. La poca cantidad de hembras de Primolius couloni en cautiverio puede deberse a: la alta susceptibilidad de hembras al estrés lo cual produce la muerte durante la captura y comercialización; los machos sean capturados en mayor medida que las hembras y que por algún motivo la cantidad de hembras en las poblaciones silvestres sean menores a la cantidad de machos.
\end{abstract}

Palabras clave: Primolius couloni, cautiverio, porcentaje de hembras, manejo reproductivo

\begin{abstract}
A total of 46 Primolius couloni individuals were studied in four different wildlife management centers in Peru: Huachipa Zoological Park, CIRES-Peru Breeding Center, Jean De Conick Breeding Center and Mundo Silvestre Breeding Center. The goal of this research was to determine the percentage of females of Primolius couloni kept in captivity with the objective of helping different wildlife management centers in developing future reproductive plans. The results show that $10.26 \%$ female Primolius couloni are kept in captivity. This mounts only to four females out of 39 successfully sexed individuals. The low amount of female Primolius couloni kept in captivity could be due to: high female stress susceptibility during capture and commercialization; males could be captured more often than females or to a lower amount of females than males in wildlife populations.
\end{abstract}

Key words: Primolius couloni, captivity, female percentage, reproductive management

\section{Introducción}

El Guacamayo cabeciazul, Primolius couloni, pertenece al orden Psitaciformes de las aves. Dentro de este orden se encuentra en la familia Psittacidae, subfamilia Psittacinae (Forshaw, 1989).

Primolius couloni puede llegar a medir 41 centímetros (Alderton, 1991) y pesar alrededor de 250 gramos (Dunning, 1993). El plumaje es predominantemente verde, siendo verde amarillento en las partes ventrales. La cabeza es completamente azul con una máscara facial gris. No existe dimorfismo sexual evidente entre machos y hembras de la especie. Por otro lado los adultos poseen el iris amarillo y patas rosadas, mientras los juveniles tienes iris oscuro y patas grises.

La distribución de esta especie abarca los países de Brasil, Bolivia y Perú. En Brasil Primolius couloni se encuentra al oeste del departamento de Acre. En
Bolivia está en la parte noroeste de los departamentos de Pando y La Paz, mientras que en Perú abarca parte de los departamentos de Loreto, San Martín, Huanuco, Pasco, Junín, Cusco, Puno; y la totalidad de los departamentos de Madre de Dios y Ucayali (Forshaw, 1989).

Existe poca información sobre Primolius couloni tanto en vida silvestre como en cautiverio. Cuando en 1998 el Dr. Nigel Collar, asesor científico de Birdlife Internacional, hizo una evaluación de la literatura mundial existente sobre psitácidos, encontró que el género Primolius (en ese momento Propyrrhura) era el que poseía menor cantidad y calidad de información publicada. De dicho género, la especie Primolius couloni era la menos estudiada (Tobias \& Brightsmith in prep.). Por lo que la información existente respecto a su historia natural y comportamiento es muy limitada o inexistente. 
A nivel nacional, hay cinco zoocriaderos y dos zoológicos registrados en el Instituto Nacional de Recursos Naturales (INRENA) que tienen individuos de Primolius couloni. De acuerdo al Decreto Supremo $\mathrm{N}^{\mathrm{o}}$ 034-2004-AG aprobado el 22 de Septiembre del 2004, para el estado peruano la especie se encuentra en situación vulnerable, lo cual indica un riesgo de extinción a mediano plazo en vida silvestre si los factores de amenaza continúan o aumentan. A nivel internacional Primolius couloni se encuentra incluida en el Apéndice I de CITES (Convención sobre el Comercio Internacional de Especies Amenazadas de Fauna y Flora Silvestres) por una propuesta hecha por el Estado Federal Alemán, apoyado por los países de Bolivia y Brasil (CITES, 2002) Según la Lista Roja de Especies Amenazadas de la IUCN (Unión Mundial de la Conservación) se encuentra en la categoría de "En Peligro” (Birdlife, 2005).

En general, en los últimos años, el estado de conservación de Primolius couloni ha cambiado a situaciones de mayor amenaza, haciendo necesaria la implementación de planes de conservación para dicha especie.

Forman parte fundamental de las estrategias de conservación de especies amenazadas los planes de conservación ex -situ, en especial aquellos que incluyen el manejo reproductivo de la especies. Muchas estrategias de manejo, en especial las reproductivas, requieren la determinación sexual de los individuos como punto de partida (Eason et al., 2001). La determinación sexual de los individuos permite conocer la cantidad de hembras y machos en la población a manejar, y de esta manera saber con cuántos núcleos reproductivos podrían contarse.

El objetivo de este estudio es determinar la cantidad de hembras y machos presentes en los zoocriaderos y zoológico evaluados, con el fin de proporcionar a los encargados de dichos lugares la información inicial para futuros planes de manejo reproductivos.

\section{Materiales y métodos \\ $\underline{\text { Lugar de estudio }}$}

El estudio se llevó a cabo en los siguientes centros de manejo de fauna silvestre: Parque Zoológico Huachipa (Lima), Zoocriadero CIRES-Perú (Puerto Maldonado), Zoocriadero Jean De Conick (Lima) y Zoocriadero Mundo Silvestre (Ica).

Metodología

Se evaluó un total de 46 individuos. 28 pertenecientes al Parque Zoológico Huachipa, cuatro al Zoocriadero CIRES-Perú, cuatro al Zoocriadero Jean de Conick y finalmente 10 del Zoocriadero Mundo Silvestre.

Para la determinación sexual se utilizó el ADN proveniente de una muestra de plumas. El uso de plumas en vez de una muestra de sangre resulta mucho menos estresante para el ave.
Para tomar la respectiva muestra de plumas, se realizó la contención física del ave. La contención física se realizó sujetando la cabeza con una mano, y las patas con la otra. La sujeción de cabeza se realizó con el pulgar y dedo medio en la mandíbula inferior, y con el dedo índice en la parte superior de la cabeza (Altman et al., 1997). Durante la sujeción de las patas se aseguró las puntas de las alas, para evitar que el ave aletee y termine exhausto. Se dejó el tórax y abdomen libres para permitir los movimientos respiratorios. Los procedimientos de contención física fueron breves para evitar el estrés excesivo, el colapso respiratorio y/o circulatorio y la muerte (Aguilar et al., 2005).

Con el uso de guantes para examinar, se retiró cuatro plumas del pecho del ave. Las plumas fueron colocadas en un sobre de papel con el número de identificación del ave. Las muestras de plumas fueron enviadas a laboratorio para la determinación sexual por ADN. Con los resultados se obtuvo el porcentaje de hembras y machos presentes en la muestra de aves sexada exitosamente.

\section{Resultados y discusión}

De las 46 aves evaluadas, se obtuvieron 35 machos, 4 hembras y 7 individuos no determinados. De las 39 aves restantes, se obtuvieron sólo cuatro hembras, lo cual representa un $10.26 \%$ de la muestra sexada exitosamente. En la Tabla 1 se muestran la distribución de machos y hembras en el zoológico y zoocriaderos.

Tabla 1. Resultados de la determinación sexual de 46 individuos de Primolius couloni mantenidos en cautiverio.

\begin{tabular}{lccc}
\hline \multirow{2}{*}{ Institución } & Machos & Hembras & $\begin{array}{c}\text { No } \\
\text { Determinados }\end{array}$ \\
\cline { 2 - 4 } & 4 & & \\
\hline $\begin{array}{l}\text { Zoocriadero } \\
\text { CIRES PERU }\end{array}$ & 3 & 1 & 1 \\
$\begin{array}{l}\text { Zoocriadero Jean } \\
\text { de Conick }\end{array}$ & 8 & 1 & 6 \\
$\begin{array}{l}\text { Zoocriadero } \\
\text { Mundo Silvestre }\end{array}$ & 20 & 2 & 7 \\
$\begin{array}{l}\text { Parque Zoológico } \\
\text { Huachipa }\end{array}$ & 35 & 4 & \\
\hline Total & & & \\
\hline
\end{tabular}

Hasta el momento se plantean tres hipótesis para explicar el bajo número de hembras en la muestra estudiada. La primera hipótesis postula que en vida silvestre la población de hembras es mucho menor que la de machos. Es decir, el porcentaje de hembras en cautiverio refleja el estado poblacional en vida silvestre. Para aceptar esta hipótesis se debe afirmar que no existe mortalidad durante la extracción hasta la comercialización o decomisos de estas aves. También, 
una especie como Primolius couloni, que presenta una baja tasa reproductiva y es presuntamente monógama al igual que la mayoría de psitácidos, debería presentar una notable disminución poblacional al tener un bajo porcentaje de hembras en su población.

La segunda hipótesis plantea que el método de captura provoca que sean más machos los atrapados. Basándose en el comportamiento reproductivo y de forrajeo de otras especies de psitácidos puede ser que los machos sean más ruidosos que las hembras al forrajear, siendo más fácil de localizar por los cazadores, o tal vez tomen más riesgos descendiendo más cerca al suelo para conseguir alimento.

En cuanto a los métodos de captura de psitácidos, se tiene registros de la zona norte del Perú. Estos registros corresponden a los métodos de captura para especies como Aratinga erytrogenys, Aratinga wagleri, Forpus coelestis y Brotogeris pyrrhopterus. Consisten en la extracción de pichones del nido, el uso de redes de neblina en dormideros y lugares de alimentación, trampas hechas con lazos de nylon, trampas con jaulas en los lugares de alimentación y caza con balines cortos, entre otros (Rosales, 1999). La única información que se tiene al respecto sobre Primolius couloni, es que probablemente son capturados en las colpas (CITES, 2002).

El uso máximo de colpa para muchas especies de psitácidos es al inicio de la época de anidamiento (Brightsmith, 2004). Durante esta época, las hembras permanecen en el nido mientras que los machos van en busca de comida y arcilla. Debido a esto, es muy probable capturar mayor cantidad de machos.

La tercera hipótesis postula que son capturados similar cantidad de machos y hembras, pero las hembras mueren durante la manipulación y transporte producto del estrés y enfermedades. En el Perú, donde cerca del 93\% de aves traficadas ilegalmente son psitácidos, existe un 43\% de tasa de mortalidad debido al estrés (Rosales, 1999) y enfermedades producida por el hacinamiento, falta de agua, falta de comida y suciedad. Puede ser que las hembras sean más susceptibles a los estresores ambientales y biológicos a los que son sometidas durante su captura, almacenamiento, transporte y comercialización.

Las tres hipótesis mencionadas anteriormente buscan explicar el bajo porcentaje de hembras en la muestra de Primolius couloni estudiada. Para poder aceptar o rechazar de manera convincente alguna de ellas hace falta estudiar la historia natural, comportamiento reproductivo y dinámica poblacional de esta especie en vida silvestre. Hay que tener en cuenta que la información existente sobre Primolius couloni es aún muy limitada.

\section{Conclusiones}

- De la muestra de Primolius couloni mantenidos en cautiverio y cuyo sexo fue determinado por análisis de ADN, se obtuvo un $10.26 \%$ de hembras, que representaban tan sólo cuatro individuos de los 39 individuos sexados exitosamente.

- Se discuten tres hipótesis para interpretar el bajo porcentaje de hembras de Primolius couloni en cautiverio: baja cantidad de hembras en vida silvestre, alta susceptibilidad de hembras al estrés lo cual produce la muerte durante la captura y comercialización, y que los machos son capturados en mayor medida que las hembras. La baja cantidad de hembras en la muestra evaluada posiblemente se debe a una combinación de las dos últimas hipótesis, pero lamentablemente no se cuenta con la suficiente información sobre la captura o comportamiento de este especie en vida silvestre para afirmar alguna de ellas.

\section{Agradecimientos}

Al Dr. Martín Gavilán de los Laboratorios Biolinks; a todo el personal del Parque Zoológico Huachipa; al Sr. Antonio Masias del Zoocriadero Mundo Silvestre; al Sr. Jean de Conick e Ing. Fabiola Enriquez del Zoocriadero Jean De Conick; y al M. Sc. Benedicto Baca del Zoocriadero CIRES-Perú.

\section{Literatura citada}

Aguilar R., Hernandez-Divers S.M. \& Hernandez-Divers S.J. 2005. Atlas de Medicina Terapéutica y Patología de Animales Exóticos. Ed Inter. Médica. Argentina.

Alderton David. 1991. The Atlas of Parrots. Ed THF Publications. Nueva Jersey, EEUU.

Altman R., Clubb S., Dorrestein G. \& Quesenberry K. 1997. Avian Medicine and Surgery. Ed WB Saunders. Filadelfia, EEUU.

Birdlife International. 2005. Species factsheet: Primolius couloni. Sitio web: http://www.birdlife.org. Último acceso 28 de Junio del 2005.

Brightsmith D. 2004. Effects of Weather on Parrot Geophagy in Tambopata, Peru. Wilson Bulletin. 116(2): 134-145.

Brightsmith D. 2005. Parrot Nesting in Southeastern Peru: Seasonal Patterns and Keystone Trees. Wilson Bulletin. 117 (3): 296-305.

CITES. 2002. Examen de las Propuestas de Enmienda a los Apéndices I y II. Prop. 12.18. Sitio web: http://www.cites.org/esp/cop/12/prop/S12-P18.pdf. Último acceso 23 Febrero del 2006.

Dunning J.B. 1993. CRC handbook of avian body masses. CRC Press. Londres, Inglaterra.

Eason D., Millar CD., Cree A., Halverson J. \& Lambert DM. 2001. A comparison of five methods for assigment of sex in the takane Porphyrio mantelli. Journal of Zoology. 253: 281-292.

Forshaw J. 1989. Parrots of the World. Ed Lansdowne. Australia.

INRENA. 2004. Categorización de especies de fauna amenazada. Decreto Supermo Nº34-2004AG, aprobado el 22 de Septiembre del 2004.

Rosales Benites M. 1999. Análisis y Alternativas de Control y Comercialización de Psitácidos del Noroeste del Perú. Tesis para obtener el Título de Magíster Scientae. UNALM. Lima, Perú. 
Tobias J. \& Brightsmith D. (in prep.) The Distribution and Conservation Status of the Blue-headed Macaw Primolius couloni.
Waugh D. com. pers. 2006. Correo electrónico enviado el 16 de Marzo del 2006.

${ }^{1}$ Universidad Nacional Agraria La Molina, aimycp@gmail.com

22Universidad Nacional Agraria La Molina, mwilliams@lamolina.edu.pe 